$10 \mid 2006$

Varia

Tournus, le castrum, l'abbaye, la ville, XIe-XIve siècles et prémices. Analyse archéologique d'un développement monastique et urbain

Thèse de doctorat de l'Université de Lyon 2, sous la direction de Jean-

François Reynaud, janvier 2006

Benjamin Saint-Jean Vitus

(2) OpenEdition

Journals

Édition électronique

URL : https://journals.openedition.org/cem/852

DOI : $10.4000 /$ cem. 852

ISSN : 1954-3093

Éditeur

Centre d'études médiévales Saint-Germain d'Auxerre

Édition imprimée

Date de publication : 15 août 2006

ISSN : 1623-5770

Référence électronique

Benjamin Saint-Jean Vitus, «Tournus, le castrum, l'abbaye, la ville, xıe-xıve siècles et prémices. Analyse archéologique d'un développement monastique et urbain », Bulletin du centre d'études médiévales d'Auxerre | BUCEMA [En ligne], 10 | 2006, mis en ligne le 09 avril 2009, consulté le 22 septembre 2022. URL : http://journals.openedition.org/cem/852 ; DOI : https://doi.org/10.4000/cem.852

Ce document a été généré automatiquement le 22 septembre 2022.

Creative Commons - Attribution - Pas d'Utilisation Commerciale - Partage dans les Mêmes Conditions 4.0 International - CC BY-NC-SA 4.0

https://creativecommons.org/licenses/by-nc-sa/4.0/ 


\section{Tournus, le castrum, l'abbaye, la ville, XIe-XIVe siècles et prémices. Analyse archéologique d'un développement monastique et urbain}

Thèse de doctorat de l'Université de Lyon 2, sous la direction de Jean-

François Reynaud, janvier 2006

\section{Benjamin Saint-Jean Vitus}

1 Le site de Tournus (Saône-et-Loire), étiré le long de la Saône, présente un exemple remarquable d'étagement topographique de vestiges antiques et médiévaux, sur $1200 \mathrm{~m}$ à peu près.

2 Au sud, l'emprise du castrum antique se lit encore très bien dans le plan parcellaire : il s'agit initialement d'une petite agglomération fortifiée du bord de Saône, traversée par la via Agrippa reliant Lyon à Chalon en suivant la rivière (le tracé de la voie se trouve à l'origine de la grand-rue du centre ville actuel). Des témoins de la muraille antique ont été conservés dans plusieurs caves du quartier.

3 Au nord s'étend l'abbaye Saint-Philibert - ou du moins ses vestiges, puisqu'elle a été sécularisée, transformée en collège de chanoines en 1627, avant d'être supprimée à la Révolution. Ce monastère bénédictin avait pris la succession, en 875 , d'un petit établissement funéraire dédié au martyr local saint Valérien, mentionné en tout cas dès le $\mathrm{VI}^{\mathrm{e}}$ siècle ; mais l'archéologie a pu attester son existence, en gros pour les $\mathrm{VI}^{\mathrm{e}}-\mathrm{VIII}^{\mathrm{e}}$ siècles, grâce à plusieurs découvertes de sarcophages à l'occasion de travaux divers, au cours $d u x^{e}$ siècle. L'abbaye médiévale, elle, se signale par un ensemble notoire de constructions des $\mathrm{XI}^{\mathrm{e}}$-XIV ${ }^{\mathrm{e}}$ siècles entourant l'ancien cloître, compris dans une enceinte dont le parcellaire conserve l'empreinte, émaillée de quelques tours. Certains éléments lui restent extérieurs, comme la chapelle Saint-Laurent, située à $400 \mathrm{~m}$ de là vers le 
nord : jusqu'à la Révolution, celle-ci se dressait isolée, au milieu des jardins et cultures qui flanquaient le rempart abbatial.

4 Entre ces deux pôles, deux églises en élévation, ainsi que des restes d'habitat dispersés de part et d'autre de la grand-rue, témoignent de l'emprise de la ville médiévale - qui englobait le noyau antique, devenu quartier du «Châtel». Si l'église dite aujourd'hui «de la Madeleine", initialement paroissiale "Sainte-Marie-du-Châtel», s'élève au cœur de l'ancien castrum, Saint-Valérien, au pied du rempart monastique, s'avère être un oratoire dépendant de l'abbaye, entouré jusqu'au $\mathrm{xv}^{\mathrm{e}}$ siècle du seul cimetière réservé aux habitants de Tournus. Ces deux édifices peuvent être datés du XII siècle. Quant aux vestiges d'architecture civile, les plus anciens repérables en élévation remontent aux XII ${ }^{\mathrm{e}}$-XIII ${ }^{\mathrm{e}}$ siècles, mais il faut retenir un ensemble conséquent du début du XIV ${ }^{e}$ siècle ou de la fin du XIII'.

Un tel dispositif laissait percevoir un modèle de développement conjoint. La taille encore mesurée de l'agglomération rendait envisageable un examen archéologique, à la fois de l'abbaye et de la ville, au moins pour les $\mathrm{XI}^{\mathrm{e}}$-XIV ${ }^{\mathrm{e}}$ siècles période charnière du Moyen Âge, tant du point de vue de l'histoire monastique, que de l'histoire de la formation des villes. Le fait que l'ensemble se soit trouvé sous la pleine autorité de l'abbaye depuis 875 (date de sa donation par le roi Charles le Chauve à la communauté vouée à saint Philibert), renforçait sa cohérence structurelle, accentuant en principe sa polarisation sur le site abbatial. La puissance du monastère, avérée par les sources écrites depuis l'époque carolingienne, ajoutait d'ailleurs à l'intérêt de cette étude - d'autant que cet établissement avait attiré l'attention jusque-là de manière sélective, au profit de l'architecture romane de son église, célébrée par maints historiens de l'art au cours du $\mathrm{xx}^{\mathrm{e}}$ siècle $^{1}$. Au reste, le site offrait la concentration de vestiges visibles la plus dense pour la période, méritant à ce titre une attention particulière.

6 Il s'avérait toutefois nécessaire, pour apprécier la réalité du basculement du centre de gravité de l'agglomération, de s'intéresser aussi au castrum initial, et au legs des périodes hautes dans le paysage pré-urbain de Tournus. En l'absence de fouille importante il est vrai, les données demeuraient peu nombreuses de ce côté : elles n'en méritaient pas moins d'être rappelées, ordonnées, et parfois corrigées.

Cette étude est donc basée sur les sources archéologiques. Mais il s'agit essentiellement de vestiges en élévations, lesquels ont fait l'objet de relevés précis en plan ou en élévation, plus ou moins détaillés selon les circonstances - ou selon les opportunités, il faut l'avouer. J'ai tout de même pu pratiquer quelques sondages et fouilles, à l'abbaye essentiellement, mais aussi sur le site du castrum, et de façon très succincte, à l'emplacement de maisons de la ville médiévale. À cela s'ajoutent les informations issues de fouilles et observations effectuées au cours $\mathrm{du} \mathrm{xx}^{\mathrm{e}}$ siècle, qu'elles aient été consignées dans des articles, ou dans des archives conservées à Tournus². J'ai cependant pris soin en parallèle, de rassembler les données textuelles, en les ordonnant autant que possible, qu'elles concernent le castrum, ou, pour la plupart, l'abbaye ou la ville médiévale ${ }^{3}$. Au besoin, elles se trouvent confrontées aux résultats des études de terrain.

Dans tout cela, les soutiens actifs, à différents niveaux, de quelques tournusiens, et des associations CIER et SAAST/GRAT ${ }^{4}$, m'auront été particulièrement précieux - tandis que les colloques et rencontres organisés sur place par le CIER, auront 
fourni l'occasion de publications intermédiaires, faisant le point sur l'avancée de mes travaux ${ }^{5}$. Enfin, je n'aurais pu mener à bien l'important travail effectué sur le terrain, en gros de 1991 à 2002, sans le concours de collègues et amis, qui m'ont prêté mainforte pour les relevés et les fouilles'.

9 Au total, la première partie de ma thèse fait d'abord le point sur les restes du castrum antique et sur l'évolution du site durant le haut Moyen Âge. Après quoi, les deux parties principales analysent en détail le site abbatial puis la ville médiévale, dont les évolutions sont présentées à chaque fois par grands états successifs, entre les $\mathrm{XI}^{\mathrm{e}} / \mathrm{XII}^{\mathrm{e}}$ siècles et le $\mathrm{XIV}^{\mathrm{e}}$ siècle. Une synthèse générale relie alors ces données, pour saisir l'articulation entre ces différents mouvements et les répercussions des modalités de développement du monastère sur la formation de ce qu'on peut appeler un bourg, jusqu'au début de la guerre de Cent ans. C'est toutefois sur le site abbatial, que l'enquête est la plus poussée, du fait de la quantité de documentation qui le concerne, de la meilleure accessibilité des vestiges, en un terrain public, mais aussi de la complexité de leur enchevêtrement.

10 Pour les périodes hautes, le principal apport concerne donc l'identification du castrum et le repérage de son enceinte à travers les caves du quartier, accompagné de quelques relevés et sondages. J'ai pu ainsi proposer une nouvelle lecture de son plan et une datation du rempart, et mettre en évidence la continuité de son occupation, de l'Antiquité tardive jusqu'au plein Moyen Âge. Au passage, on notera la réalité de travaux sur la muraille vers la fin du $\mathrm{IX}^{\mathrm{e}}$ siècle. Quant à l'établissement voué à saint Valérien à l'emplacement de la future abbaye, ses origines hautes restent à découvrir ; on peut au moins déduire des données de fouille, que son emprise était plus étroite que le site actuel, et qu'il se concentrait sans doute au sommet de la butte, entouré d'une nécropole matérialisée par les sarcophages des $\mathrm{V}^{\mathrm{e}}$-VIII ${ }^{\mathrm{e}}$ siècles déjà découverts. Les dernières observations y trahissent aussi d'importants terrassements, liés à un remaniement d'ensemble des lieux, qui doit suivre la donation de 875 .

11 Pour l'abbaye des $\mathrm{XI}^{\mathrm{e}}$-XIV ${ }^{\mathrm{e}}$ siècles, mon premier travail de terrain aura été alors, de démêler l'écheveau des reconstructions multiples, associant certaines d'entre elles à des données de fouille dans une même chronologie relative, pour proposer au final sept états successifs, que j'ai datés entre le premier quart du xI siècle et le milieu du XIV ${ }^{e}$. Les plans correspondant englobent à chaque fois l'emprise actuelle de l'église abbatiale et du cloître, mais aussi, selon les cas, des bâtiments établis sur le tracé de l'enceinte, et même la petite chapelle Saint-Laurent. Dans ce cadre et pour chaque période ainsi définie, sont analysées les fonctions des bâtiments et des espaces extérieurs et leurs relations entre eux, ainsi que les intentions que ces aménagements éclairent.

Pour la ville enfin, et pour la même période, l'étude plus ou moins détaillée des deux églises subsistantes et d'une trentaine de maisons conservées, ou d'indices d'habitat repérés en fouille ou en élévation, combinée à quelques données historiques concernant la topographie et les paroisses, permet de définir, de manière plus lâche, deux étapes majeures de l'évolution urbaine, au XII ${ }^{\mathrm{e}}$ et dans la première moitié du XIII ${ }^{\mathrm{e}}$ siècle, puis dans le dernier tiers du XIII $^{e}$ et la première moitié du XIV $^{e}$ siècle. L'interprétation se fait ici dans deux directions : par rapport aux formes, fonctions et significations propres à l'habitat, et par rapport aux modalités d'évolution topographique, dans l'optique de la relation avec le pouvoir abbatial sous ses différentes expressions. 
Dans le détail de l'étude archéologique, un effort important a été engagé pour préciser les datations : notamment, selon les critères absolus d'analyses en laboratoire.

Là où des fouilles ont été possibles, les chronologies relatives révélées par la stratigraphie - et se poursuivant parfois dans le suivi des élévations à l'abbaye - ont été confortées par des datations par le radiocarbone ${ }^{7}$. Quelques rares monnaies en place en stratigraphie, et surtout une céramique abondante, quoique toujours très fragmentée, ont été également exploitées. C'est d'après quelquestessons recueillis dans ses fondations, qu'a pu être avancée une datation de la muraille du castrum,en gros entre 250 et $350^{8}$. Et la continuité chronologique existant d'une fouille à l'autre à travers la ville permet de suivre l'évolution typologique des mobiliers à Tournus, pratiquement de la fin de l'Antiquité à la fin du Moyen Âge'.

Pour les élévations, c'est aussi le nombre d'analyses dendrochronologiques qui constitue l'un des points forts de ce travail - grâce à la collaboration bienveillante de Georges-Noël Lambert (CNRS, laboratoire de chrono-écologie du quaternaire, Besançon). Il s'est agi là d'un travail de longue haleine. La mise en place d'un référentiel propre à Tournus pour la période $\mathrm{x}^{\mathrm{e}}$-XIV ${ }^{\mathrm{e}}$ siècle nous a d'ailleurs conduit, pour étoffer le corpus et saisir les particularités des milieux locaux, à susciter des prélèvements sur plusieurs autres sites environnants, de Saint-Martin de Laives ou Chapaize (églises du $\mathrm{XI}^{\mathrm{e}}$ siècle) à Saint-Gengoux-le-National ou Germolles (maisons et château du XIV ${ }^{\mathrm{e}}$ siècle).

À 1 ces données objectives s'ajoute néanmoins, pour l'abbaye et les églises conservées du bourg, la prise en compte de l'évolution du décor sculpté, du XI ${ }^{\mathrm{e}}$ au XIV siècle, déjà étudié par plusieurs historiens de l'art ${ }^{10}$ : dans le premier cas, j'ai simplement reclassé les sculptures en fonction de leur position en chronologie relative, soulevant ici et là quelques questions particulières.

Enfin, dans la mesure où j'avais affaire à des maçonneries pour l'essentiel, une grosse partie de mon travail a consisté à identifier et classer les modes de mise en œuvre, par l'analyse détaillée des appareils, et dans une moindre mesure, par les traces d'outils observables sur les pierres de taille. M'appuyant sur les données précédentes, j'ai tenté d'en retirer une chronologie de l'évolution des modes de construction aux $\mathrm{Xl}^{\mathrm{e}} \mathrm{XIV}^{\mathrm{e}}$ siècle : valable pour tout le milieu tournusien, celle-ci peut désormais s'appuyer sur un corpus-témoin de relevés et photos de détail.

8 En fin de compte, ce travail sur la chronologie a permis, une fois débrouillée la genèse du site avant l'an mil, et à partir des «états » successifs caractérisant chaque site, une nouvelle lecture du développement abbatial et de la croissance du bourg, du Xi ${ }^{e}$ au XIV ${ }^{e}$ siècle.

Pour l'abbaye, l'accent est mis sur l'importance de la restructuration engagée dans le second quart $\mathrm{du} \mathrm{xl}^{\mathrm{e}}$ siècle. Soulignées par Jacques Henriet à propos de la seule église Saint-Philibert, les transformations de cette période, décelées de façon plus large à travers le monastère, présentent un caractère radical, qui traduit un projet concerté, mû par une volonté réformatrice - où transpire l'influence de Cluny. Sa réalisation se poursuit jusqu'aux alentours de 1100.

De fait, les dispositions apparues comme héritées du $\mathrm{x}^{\mathrm{e}}$ siècle ou des alentours de l'an mil laissaient deviner d'abord une petite église flanquée de bâtiments au sud, qui encadraient peut-être un cloître ; à l'ouest, ceux-ci étaient précédés d'une construction à vocation funéraire, à poteaux de bois et sol de béton. Un réseau d'oratoires secondaires maillait sans doute déjà le territoire proche, à l'image de la chapelle Saint- 
Laurent, isolée au nord du monastère. Mais la reconstruction de la crypte et du sanctuaire de l'abbatiale amorçait, dès les années 1015/1020, la reprise de l'ensemble. Toutefois, celle-ci ne révèle un programme abouti qu'à partir du second quart $\mathrm{du} \mathrm{XI^{ \textrm {e } }}$ siècle, lorsque sous l'impulsion de l'abbé Ardain, on assiste non seulement à une réfection ambitieuse de la grande église, comprenant notamment à son extrémité ouest, l'adjonction d'une "galilée " à deux niveaux superposés, mais aussi à la réorganisation de l'ensemble claustral - qui entraîne la suppression du bâtiment funéraire occidental. Le nouvel agencement implique une redéfinition des espaces à travers le monastère, qui les ordonne plus strictement selon une bipolarité est/ouest, traduite en sacré/profane et sans doute en religieux/laïc, rejetant probablement les zones d'inhumations vers le nord et l'est, pour réserver les secteurs méridionaux et occidentaux aux activités de service et peut-être d'accueil. Cet élan se poursuit jusqu'à la fin du siècle, même si le rythme des travaux ralentit dans sa seconde moitié : autour de 1100, la réfection de l'église s'achève, alors que la reprise de l'aile occidentale clôt la restructuration du cloître. Les progrès des techniques constructives ont suivi l'avancée du chantier.

Aatdelà, les travaux des $\mathrm{XII}^{\mathrm{e}}$ et $\mathrm{XIII}^{\mathrm{e}}$ siècles ne remettent pas en cause fondamentalement ce dispositif. Ils sont pourtant de grande ampleur, concernant presque tous les espaces du monastère, qu'ils rhabillent ou embellissent, quand ils ne les reconstruisent pas, à la façon de l'aile méridionale du cloître, dotée au XII siècle d'un vaste réfectoire flanqué d'une tour à la jonction du vieux dortoir, et précédé à l'ouest d'une monumentale cuisine à plan centrée, découverte à la fouille. De ce point de vue, le XII ${ }^{e}$ siècle offre la vision la plus complète, la plus précise, la plus quotidienne et la plus faste, du site de l'abbaye - tandis que la qualité de la mise en œuvre mérite d'être soulignée. Sur sa fin, malgré un possible ralentissement d'activité, les difficultés financières avérées n'empêchent pas quelques réalisations de prestige. La première moitié du XIII ${ }^{\mathrm{e}}$ siècle, elle, marque encore fortement l'ensemble du site, à une époque où les abbés affirment toujours leur puissance. C'est dans ces périodes sans doute, qu'est mise en place la mosaïque dégagée dans le déambulatoire du chœur de l'abbatiale : elle figure la succession des mois de l'année et des signes du zodiaque ${ }^{11}$. Aussi faut-il attendre la fin du XIII ${ }^{e}$ siècle et le début du XIV ${ }^{e}$, pour constater un clair tarissement des travaux. Ceux-ci se concentrent d'ailleurs sur les fortifications aux premiers échos de la guerre de Cent Ans. Mais dans le même temps, une modification se fait sentir dans l'équilibre des relations entre religieux et laïcs.

En parallèle à cette évolution, le bourg semble s'être développé à partir du noyau urbain de l'ancien castrum et jusqu'à l'abbaye le long de l'ancienne voie romaine, autour d'églises et d'oratoires sous la tutelle des moines - voire du cimetière qui jouxte l'abbaye, celui des Tournusiens. Le XII ${ }^{\mathrm{e}}$ siècle s'affirme ici comme une période charnière, puisque on y saisit le débordement de l'enceinte antique du castrum, alors que tous les édifices religieux connus sont reconstruits - assurément sous l'impulsion du monastère. À cette période aussi remontent les plus anciens témoignages attestés d'un habitat bourgeois.

Jusqu'au milieu du XIII ${ }^{\mathrm{e}}$ siècle, les quelques vestiges reconnus sont ceux de grandes maisons de pierre sur cellier de rez-de-chaussée, dont deux au moins connaissaient une partie centrale élancée en forme de tour ${ }^{12}$. Ces demeures disposaient dans les grandes salles des étages, du confort d'une cheminée dont le conduit s'affirmait à l'extérieur, sous forme d'un solide massif vertical en débord de façade ; la 
circulation d'un niveau à l'autre s'y faisait peut-être au moyen de galeries de bois extérieures. Situées en des endroits privilégiés de la ville, elles hébergeaient vraisemblablement des fidèles ou alliés du monastère, assurant un relais de l'autorité monastique sur les Tournusiens, et formant peut-être, à leur tour, autant de pôles susceptibles d'attirer le développement urbain.

À la fin du XIII et au XIV ${ }^{e}$ siècle, des exemples bien plus nombreux suggèrent la quasibanalisation d'une forme d'habitat bourgeois tourné sur la rue, sur laquelle ouvrent à la fois le large portail de la salle du rez-de-chaussée, à vocation probable de magasin ou d'atelier, et l'escalier droit qui conduit à un étage indépendant destiné au logement. On saisit désormais la formation de fronts de rue, tandis que de nouveaux espaces semblent colonisés, esquissant pour la première fois la trame continue du centre ville actuel. De nouvelles formes de confort domestique se font jour, principalement à travers la généralisation des fenêtres géminées sur rue, souvent très ornées sur l'extérieur, munies de coussièges et parfois de volets de bois; le modèle gagne même une ancienne maison en hauteur $\mathrm{du} \mathrm{XII}^{\mathrm{e}}$ siècle.

Ainsi, ce développement conjoint traduit d'abord une impulsion monastique forte. Celle-ci est suscitée par l'élan réformateur identifiable au xI ${ }^{\mathrm{e}}$ siècle à Saint-Philibert. La puissance de l'abbaye aux $\mathrm{XII}^{\mathrm{e}}$-XIII ${ }^{\mathrm{e}}$ siècle entraîne sans doute la ville dans son sillage. Mais cette poussée urbaine ne se fait pas sans contrôle : la tutelle du monastère se fait sentir derrière les reconstructions d'églises, l'implantation du cimetière, ou de manière moins directe, derrière l'expression dominatrice de quelques maisons en hauteur. Pourtant, à partir de la seconde moitié du XIII ${ }^{\mathrm{e}}$ siècle s'affirme une autre dynamique : elle est plus proprement laïque, et bourgeoise.

\section{NOTES}

1. Principales études concernant l'ancienne église abbatiale Saint-Philibert : J. VIREY, « Les dates de construction de Saint-Philibert de Tournus ", in Bulletin monumental, 67 (1903), p. 556-561; IDEM, «Les différentes époques de construction de Saint-Philibert. Étude préliminaire », in H. CURE, Saint-Philibert de Tournus. Guide historique et descriptif de l'Abbaye, Paris, 1905 (rééd. SaintSeine-l'Abbaye, 1984, p. 17-43) ; J. VALLERY-RADOT, Saint-Philibert de Tournus, Paris, 1955 ; F. LESUEUR, "Hypothèses sur la construction de Saint-Philibert de Tournus ", in Mélanges René Crozet, t. 1, 1966, p. 215-223 ; C.-E. ARMI, Saint-Philibert at Tournus and wall systems of first romanesque architecture, Dissertation, New York, Columbia University, 1973 (dactylographié) ; C.-E. ARMI, "The Nave of Saint-Philibert at Tournus ", in Journal of the Society of architectural Historians, 60/1 (mars 2001), p. 46-67 ; J. HENRIET, «Saint-Philibert de Tournus. Histoire, critique d'authenticité, étude archéologique du chevet (1009-1019)», in Bulletin monumental, 148/3 (1990), p. 229-316; IDEM, « Saint-Philibert de Tournus. L'œuvre du second maître : la galilée et la nef », in Bulletin monumental, 150/2 (1992), p. 101-164; C. SAPIN « Saint-Philibert et les débuts de l'architecture romane en Bourgogne ", in J. THIRION (dir.), Saint-Philibert de Tournus. Histoire. Archéologie. Art, actes du colloque du Centre international d'études romanes de Tournus des 15-19 juin 1994, Tournus, 1995, p. 215-230; IDEM, «La pierre et le voûtement, innovation dans les techniques de 
construction des églises de Bourgogne au XIe siècle ", in P. BECK (dir.), L'innovation technique au Moyen Âge, Paris, 1998, p. 179-185 ; IDEM, «D’Auxerre à Cluny, le dossier archéologique des premières avant-nefs et galilées ", in C. SAPIN (dir.), Avant-nefs et espaces d'accueil dans l'église entre le IVe et le XIIe siècle, Paris, 2002, p. 398-413; K. KRÜGER, "Tournus et la fonction des galilées en Bourgogne ", in C. SAPIN (dir.), Avant-nefs et espaces..., ibid., p. 414-423. Depuis les travaux érudits des historiens locaux des Xvie-XviIIe siècles, les principales études historiques récentes reprenant les sources sur le sujet sont celles de J.-P. ANDRIEUX, La communauté de Saint-Philibert de 677 à l'an Mil. Contribution à l'étude des origines de la personne juridique, thèse dactylographiée de doctorat en droit, Université de Paris-II/Panthéon-Assas, 1993 et surtout I. CARTRON-KAWE, Peregrinationes et congregatio sancti Filiberti. De l'Aquitaine à la Provence: la genèse du réseau monastique de SaintPhilibert, du IXe au xıe siècle, thèse dactylographiée de doctorat en histoire et archéologie médiévales, sous la direction de M. Fixot, Université de Provence, Aix-en-Provence, 1998 ; cf. aussi D. IOGNA-PRAT, « Un texte hagiographique épineux : la translatio sancti Valeriani », in J. THIRION (dir.), Saint-Philibert de Tournus..., op. cit., p. 27-40 et É. PALAzzo, « La liturgie autour de Tournus au Moyen Âge ", in J. THIRION (dir.), Saint-Philibert de Tournus..., ibid., p. 87-104.

2. Il s'agit essentiellement des travaux des érudits locaux J. Martin, G. Jeanton et A. Bernard. Voir entre autres J. MARTIN, « Découvertes archéologiques dans les dépendances de l'église abbatiale de Tournus", mémoire présenté au Congrès archéologique de France, Mâcon, 1899, in Annales de l'Académie de Mâcon, 3e série, 5 (1900), p. 136-144 et "Étude rétrospective sur les anciens bâtiments de l'abbaye de Tournus", in Annales de l'Académie de Mâcon, 3e série, 5 (1900), p. 255-287; G. JEANTON, "L'ancienne ville romaine de Tournus et son Castrum ", in Bulletin archéologique du Comité des travaux historiques et scientifiques, 1920, p. 155-181 et «L'ancienne ville romaine de Tournus et son enceinte » in Bulletin de la Société des amis des arts et des sciences de Tournus, 1921, p. 131-165 ; A. BERNARD, « Dictionnaire historique et topographique des rues, places et promenades de la ville de Tournus ", in Bulletin de la Société des amis des arts et des sciences de Tournus, 1911, p. 37-128 et 1912, p. 17-92.

3. Principales études historiques récentes concernant Tournus ou son abbaye au cours du Moyen Âge: G. DUBY, La société aux XIe et XIIe siècles dans la région mâconnaise, Paris, 1953 (rééd. 1971 et 1988); F. AUMONIER, La seigneurie de l'abbaye Saint-Philibert sur la ville de Tournus, mémoire dactylographié de DESS d'histoire, sous la direction de J. Richard, Université de Bourgogne, Dijon, 1970 et F. BRACCONI, «La seigneurie de l'abbaye Saint-Philibert sur la ville de Tournus ", in Bulletin de la Société des amis des arts et des sciences de Tournus, 1977, p. 27-103; voir aussi J.-P. ANDRIEUX, D IOGNA-PRAT, É. PALAZZO, I. CARTRON-KAWE et K. KRÜGER cités dans la note 1.

4. "Centre international d'études romanes" et "Société des amis des arts et des sciences de Tournus / Groupe de recherches archéologiques du Tournugeois»: mes principaux interlocuteurs (et soutiens) ont été, respectivement, Melle Marguerite Thibert et M.Jean Duriaud. Il faut leur adjoindre Mme Anne-Françoise Pataille, longtemps conservatrice de la bibliothèque municipale.

5. B. SAINT-JEAN VITUS, «Les bâtiments claustraux de Saint-Philibert au Moyen Âge », in J. THIRION (dir.), Saint-Philibert de Tournus..., op. cit., p. 231-252 et fig. 1 de la page 3 de la couverture ; IDEM, «Tournus, du castrum antique au bourg médiéval: paysage monastique et développement urbain ", in J. THIRION (dir.), Saint-Philibert de Tournus..., ibid., p. 383-396; IDEM, "L'abbaye de Tournus autour de l'an Mil », in Les actes des rencontres de Tournus. Histoire. Littérature. Archéologie. Art, 14 et 15 septembre 2000, Tournus, 2002, p. 66-75; IDEM, «La mosaïque du déambulatoire du chœur à Saint-Philibert de Tournus", in Décor retrouvé à Saint-Philibert, 2004, p.33-54. L'exposition « 30 ans de recherches archéologiques en Saône-et-Loire » avait également fourni l'occasion d'une présentation intermédiaire de mes travaux, cf. « Le castrum de Tournus ", in 30 ans d'archéologie en Saône-et-Loire,Mâcon, 1996, p. 244 et "Tournus, la formation d'une ville à l'ombre de l'abbaye ", in 30 ans d'archéologie..., ibid., p. 368-376. À propos de certaines des maisons 
médiévales de Tournus, cf. aussi B. SAINT-JEAN VITUS, «Maisons-tours et maisons de bourgs. Des parentés dans l'habitat noble entre milieux urbain et rural en Bourgogne, xIIe-XIve siècle. À propos de trois maisons de Tournus (Saône-et-Loire) à corps principal surélevé ", in Pages d'archéologie médiévale en Rhône-Alpes, actes de la 4e rencontre Rhône-Alpes d'archéologie médiévale, Lyon, 1997, p. 97-112.

6. Parmi bien d'autres, je citerai au moins Olivier Juffard, alors dessinateur en archéologie et topographe, auquel je dois beaucoup des relevés de terrain qui m'ont servi pour ma thèse, effectués pour la plupart entre 1991 et 1997, cf. O. JUFFARD et B.SAINT-JEAN VITUS, "Quelques maisons bourgeoises du Tournus médiéval », in J. THIRIon (dir.), Saint-Philibert de Tournus..., op. cit., p. 397-426.

7. Effectuées par le Centre de Datation par le Radiocarbone de Lyon-Villeurbanne (CNRS/ Université Claude Bernard Lyon 1 : Claude Évin, Christine Oberlin).

8. L'identification et la datation de ces tessons est due à Sylvie Mouton-Venault, spécialiste de la céramique de l'Antiquité tardive en Bourgogne.

9. Je dois à Emmanuel Poil, spécialiste de la céramique médiévale en Bourgogne, d'avoir établi la typo-chronologie du mobilier céramique issu de toutes ces fouilles.

10. Voir principalement M. et C. DICKSON, Les églises romanes de l'ancien diocèse de Chalon, Mâcon, 1935 ; J. VAlLERY-RADOT, Saint-Philibert de Tournus, op. cit.; É. vergnOlle (dir.), « Recherches sur quelques séries de chapiteaux romans bourguignons ", in L'Information d'histoire de l'art, marsavril 1975, p. 55-79 ; J. HENRIET, «Saint-Philibert de Tournus. Histoire... », op. cit., p. 229-316 et IDEM, « Saint-Philibert de Tournus. L'œuvre... », op. cit., p. 101-164; C. SAPIN, « Saint-Philibert et les débuts de l'architecture romane en Bourgogne », in J. THIRION (dir.), Saint-Philibert de Tournus..., op. cit., p. 215-230 ; C.-E. ARMI, «The Nave of Saint-Philibert... », op. cit., p. 46-67 et É. VERGNOLLE, "Saint-Philibert de Tournus : le remaniement des parties orientales au début du XIIe siècle et son décor sculpté ", in Le décor retrouvé à Saint-Philibert de Tournus. Regards sur la mosaïque médiévale, actes du colloque du Centre international d'études romanes de Tournus des 18-19 septembre 2003, Tournus, 2004, p. 140-175.

11. Cf. B. SAINT-JEAN VITUS, « La mosaïque du déambulatoire du chœur... », n. 5.

12. Sur ce sujet, cf. SAINT-JEAN VITUS « Maisons-tours et maisons de bourgs... », n. 5 .

\section{INDEX}

Mots-clés : castrum, abbaye, ville

Index géographique : France/Tournus 